\title{
Adaptación y Validación de Instrumentos de Medida del Compromiso Escolar y los Factores Contextuales en Estudiantes de Niveles Iniciales de Educación en Chile
}

\author{
Adaptation and Validation of Instruments for the Measurement of School \\ Engagement and Contextual Factors in Students of Primary Levels of Education in \\ Chile
}

\author{
Belén Sotomayor ${ }^{1}$, Laura Lara ${ }^{2}$ y Mahia Saracostti ${ }^{3}$
}

\begin{abstract}
Resumen
El objetivo del estudio fue adaptar y validar cuestionarios de medición del Compromiso Escolar y de Factores Contextuales para su utilización con estudiantes de niveles iniciales de enseñanza en Chile. Tras la adaptación de los instrumentos, mediante juicios de expertos y grupos de discusión con estudiantes, fueron aplicados a 420 estudiantes que cursaban quinto y sexto básico en Chile. Los análisis factoriales confirmatorios mostraron que los instrumentos se ajustaron a las configuraciones originales. Además, ambos cuestionarios mostraron adecuados índices de consistencia interna, y sus puntuaciones correlacionaron de la forma esperada con los resultados académicos y de asistencia de los/as estudiantes.
\end{abstract}

Palabras clave: compromiso escolar, contexto, autoinforme, ajuste, estudiantes

\begin{abstract}
The objective of the study was to adapt and validate questionnaires for measuring School Engagement and Contextual Factors for use with students of initial levels of education in Chile. After the adaptation of the instruments, through expert judgments and discussion groups with students, the questionnaires were applied to 420 students who were in fifth and sixth grade in Chile. Confirmatory factor analysis showed that the instruments maintained the same factors of the original version. In addition, both questionnaires showed adequate internal consistency indexes, and as expected, their scores correlated with students' academic results and school attendance.
\end{abstract}

Keywords: school engagement, context, self-report, adjustment, students

\footnotetext{
1 Máster en Educación. Estudiante Ph.D. Educación, Universidad de Almería, España. Investigadora. Universidad de la Frontera. Universidad de la Frontera, Avenida Francisco Salazar, 01145, Temuco, Chile. Tel.: +56 952531464. Correo: mbsotomayor@uc.cl

${ }^{2}$ Laura Lara. Doctora en Psicología. Profesora Titular. Universidad Autónoma de Chile, 5 poniente 1670, Talca, Chile. Tel.: +56712735770. Correo:1larav@uautonoma.cl

${ }^{3}$ Doctora en Social Welfare. Profesora Titular de la Escuela de Trabajo Social de la Facultad de Ciencias Sociales. Directora de la Cátedra UNESCO Niñez, Juventud, Educación y Sociedad. Universidad de Valparaíso. Universidad de Valparaíso, Blanco 951, Valparaíso, Chile. Tel.: +56998293161. Correo: mahia.saracostti@uv.cl
} 


\section{Introducción}

La educación es un derecho fundamental y tiene un efecto multiplicador en el ejercicio de los derechos Humanos (Van Dijk, 2012). El derecho a la educación puede concebirse como un proceso continuo, con avances y retrocesos, cuyos contenidos son objeto de disputa y de movilización permanente. Asimismo, es concebido como un fenómeno complejo que se registra en mayor o menor grado en todos los países, afectado por otros fenómenos contemporáneos (desigualdad, pobreza, exclusión, multiculturalismo, migración) cuyas consecuencias, además, varían de acuerdo con los grupos sociales, etarios, étnicos, religiosos, etcétera (Zurita, 2011). Desde una mirada sistémica, cuando un niño, niña o adolescente deja de asistir a la escuela sin haber concluido el ciclo escolar o todos los grados de la educación básica obligatoria, quien estaría en falta sería, principalmente, el sistema escolar (Van Dijk, 2012).

El Compromiso Escolar se posiciona como un elemento clave para la garantía del derecho a la educación debido a que posee el potencial de predecir trayectorias educativas positivas (Appleton, Christenson, \& Furlong, 2018; Furlong \& Christenson 2008; Hazel, Vazirabadi, \& Gallagher, 2013; Lara et al., 2018; Molinari \& Sánchez-Rosas, 2018). Además, contribuye positivamente al desarrollo del aprendizaje y a la finalización de los estudios de los/as estudiantes (Molinari et al., 2018; Rumberger \& Rotermund, 2012). Algunos estudios han demostrado que el Compromiso Escolar está influenciado por el entorno el que impacta en los aprendizajes de los y las estudiantes más que cualquier otro concepto. Es además un mediador entre el ambiente y el aprendizaje del/a estudiante, sin embargo, esta acción mediadora, no se mostraría explícitamente, especialmente en el contexto de la educación secundaria (Shernoff, Ruzek, \& Sinha, 2017).

Según Shernoff (2013) el Compromiso Escolar se define como una simultánea experiencia de concentración, interés y disfrute intenso del estudiantado. En esa misma línea, sería considerado como una experiencia de aprendizaje, teniendo un valor en sí mismo, y no tan solo como un predictor (Sandoval-Muñoz,
Mayorga-Muñoz, Elgueta-Sepúlveda, SotoHiguera, Viveros-Lopomo, \& RiquelmeSandoval, 2018). Al mismo tiempo es un factor importante para obtener trayectorias educativas exitosas (Fredricks, Blumenfeld, \& Paris, 2004; Hazel et al., 2013), y que de acuerdo con los aportes de Alrashidi, Phan y Ngu (2016), es un término complejo que enfatiza diversos patrones de motivación, cognición y comportamiento de los y las estudiantes.

El Compromiso Escolar puede concebirse como una metaconstrucción (Bond, Buntins, Bedenlier, Zawacki-Richter, \& Kerres, 2020; Fredricks et al., 2004; Kahu, 2013). Entendiéndose metaconstrucción, según Fredricks et al. (2004), como el potencial de ser un constructo multidimensional, conformado por distintas, pero integradas, dimensiones que interactúan y se influyen entre sí. La literatura científica sobre el Compromiso Escolar ha diferenciado tres componentes: conductual, afectivo y cognitivo (Appleton et al., 2008; Archambault \& Vandenbossche-Makombo, 2014; Fredricks et al., 2004; Gutiérrez, Tomása, Romero, \& Barricac, 2016; Hazel et al., 2013; Lam et al., 2014).

Primero, el compromiso conductual hace referencia a la participación de los y las estudiantes en diferentes actividades, lo cual se relaciona con el rendimiento académico y la prevención del abandono escolar (Fredricks et al., 2004). Entendiendo a la participación escolar, a su vez, como un conjunto de prácticas que fomentan la expresión de opiniones y el involucramiento en los procesos de decisión democrática de los y las estudiantes (Pérez-Salas, Sirlopú, Cobo-Rendón, $\&$ Awad, 2018). Segundo, el compromiso afectivo involucra las reacciones positivas y negativas de maestros y estudiantes; fomentando un vínculo con la escuela (Fredricks et al., 2004). Por último, el compromiso cognitivo se basa en la idea de inversión e incorporación de la voluntad de ejercer el esfuerzo necesario para comprender ideas y desarrollar habilidades complejas (Fredricks et al., 2004).

Además, el Compromiso Escolar es concebido como un fenómeno biopsicosocial y responde a contextos ambientales dentro de una trayectoria de desarrollo (Hazel, Wonner, \& Jack, 2008), aludiendo al estado de involucramiento de un 
estudiante con su entorno escolar (MirandaZapata, Lara, Navarro, Saracostti, \& de-Toro, 2018). La creación de un compromiso significativo requiere poner atención a una variedad de contextos, enseñanzas, factores de desarrollo e interpersonales, más allá de la preocupación por resultados educativos (Shernoff, 2012). Las definiciones del concepto, en general, no especifican un contexto, lo que permitiría comprender que el Compromiso Escolar ocurre dentro de una escuela y debería desarrollarse, también, en contextos más informales (SandovalMuñoz et al., 2018). Además, se ha demostrado la importancia del Compromiso Escolar por sus significativas relaciones con variables del contexto escolar (Christenson et al., 2012; Gutiérrez et al., 2016). Los Factores Contextuales influyen, a su vez, en las tasas de graduación de la escuela secundaria (Zaff et al., 2017).

Lo anterior, ofrece esperanzas a educadores y educadoras, al considerar que el Compromiso Escolar es influenciado por el medio ambiente y, por lo tanto, es maleable (Fredricks et al., 2004; Shernoff, 2012). Por ende, las escuelas pueden modificar sus contextos para aumentar el compromiso de los y las estudiantes. En ese sentido, comprenderse como maleable significaría que es plástico, que puede ser cambiado y aumentado (Hazel et al., 2013). Por consiguiente, el Compromiso Escolar está influenciado por contextos que son modificables, como la relación entre pares, la relación con el profesorado y la influencia de la familia en la escuela (Ansong, Okumu, Bowen, Walker, \& Eisensmith, 2017; Gutiérrez et al., 2017; Rodríguez-Fernández et al., 2016; Miranda-Zapata et al., 2018; Shernoff, 2012). Adicionalmente, los Factores Contextuales son expresados en vínculos emocionales del estudiantado con los otros actores de su proceso educativo (Sandoval-Muñoz et al., 2018).

El Compromiso Escolar es un predictor estadísticamente significativo del abandono escolar (Appleton et al., 2008; Fredricks et al., 2004; Li, Gao, \& Sha, 2020; Rumberger \& Rotermund, 2012; Van Dijk, 2012). Sin embargo, la problemática de la deserción escolar afecta al conjunto de la sociedad chilena, como consecuencia de la situación de marginación que vive gran parte de las y los/as ciudadanos y la inequidad que se arrastra $y$ que se mantiene dramáticamente vigente (Abraham \& Lavín, 2017). Reconociendo al abandono escolar como el signo más visible de un ciclo gradual de desconexión (Furlong et al., 2008). El abandono escolar temprano es uno de los principales problemas para el éxito de las políticas educativas (García, 2016). Este afecta a los y las estudiantes, sus familias y a los gobiernos (Sagar \& Mehta, 2018). Asimismo, se pueden visualizar problemáticas como el absentismo escolar (Li et al., 2020; Rumberger \& Lim, 2008; Sagar et al., 2018), comportamientos de riesgo, bajo rendimiento en el estudio, maternidad adolescente, baja adaptación a normas de convivencia, bajo nivel socioeconómico, falta de apoyo de la familia y desmotivación académica, entre otros (Sagar et al., 2018). En cuanto a la desmotivación académica, se ha demostrado que el interés y la ansiedad académica no afectan al Compromiso Escolar directamente, sino a través de la autoeficacia percibida por el estudiantado (Li et al., 2020). Siendo un factor importante en la estimulación de la motivación y la confianza para completar sus tareas académicas (Li et al., 2020).

De acuerdo con lo que señalan Rumberger et al. (2017), los y las estudiantes de sexto grado en Filadelfia, Estados Unidos, presentaron indicadores de riesgos, como baja asistencia, evaluaciones insatisfactorias del comportamiento y reprobación de algunas materias, desencadenando de un 10 a $20 \%$ de posibilidades de graduarse a tiempo que su curso. Además, los resultados de un estudio realizado en Angola y República Dominicana (Veiga, 2013), reportan relaciones estadísticamente significativas a partir de la orientación de metas de los/as alumnos/as y el clima motivacional del aula. Se suman a estas evidencias, el compromiso y estrecho vínculo que estudiantes establecen con su escuela y que dan cuenta de su alto nivel de satisfacción escolar (Gutiérrez et al., 2016).

Una investigación del Compromiso Escolar en edad temprana (Ladd \& Dinela, 2019) en Estados Unidos, asociado a modelos de estabilidad mediante un análisis de ruta en el primer, segundo y tercer grado, destaca la capacidad predictiva del Compromiso Escolar Emocional con el Compromiso Escolar Conductual durante los grados primarios y viceversa. En el estudio realizado por Van Dijk (2012), donde realizó 
entrevistas a niños/as y adolescentes, dio a conocer que una cuarta parte de los y las participantes habían repetido y, de estos, 26\% había reprobado el primer grado de primaria. Durante las entrevistas también se consultó a los y las participantes con respecto al principal motivo para dejar la escuela y se concluye que sería un fenómeno multi-causal, donde una situación desencadena otra y conduce a cierta toma de decisiones.

En base a aquellos antecedentes, la deserción escolar en la primaria es un fenómeno latente que contrarresta a gobierno, escuelas, familias y a los/as propios/as estudiantes; interpelando y cuestionando las condiciones en las que se está generando el problema educativo (Forero \& Lugo, 2017). De acuerdo con evidencia empírica, da cuenta del aumento de estudiantes en el primer ciclo de educación básica que tienden a abandonar tempranamente la institución educativa formal (Julio, 2017), que estaría afectando además la ruta de los 13 años de obligatoriedad escolar en Chile.

A pesar de que la tasa de incidencia global de la deserción en Chile disminuyó del 2,3\% en 2012 al 1,4\% en 2018 (MINEDUC, 2020), en base a las estimaciones realizadas por el Centro de Estudios MINEDUC (2018 como se citó en MINEDUC, 2020), la tasa de deserción escolar global fue del $1,4 \%$, mientras que la tasa de incidencia regular fue del 2,4\%. Además, se estima que en Chile la tasa de prevalencia global de abandono escolar entre $\operatorname{los} 6$ y 21 años es de un 8,99\%, lo que correspondería a 358.946 jóvenes chilenos (CIAE, 2019). Cabe mencionar que entre las etapas de niñez y adolescencia se ven aumentadas las posibilidades para llegar a un nivel de compromiso cognitivo superior, estando asociado a beneficios académicos y a la realización de tareas cognitivas y socioemocionales (Christenson et al., 2012). Además, es necesario considerar que América Latina presenta elevadas tasas de deserción a temprana edad (Forero et al., 2017). En este sentido, la deserción escolar temprana es un fenómeno complejo, sistémico, de carácter pedagógico, interaccional, intersubjetivo y cultural, porque refiere a procesos de formación de personas que provienen de contextos históricos situados (Julio, 2017). Además, lo y las estudiantes que dejan inconclusas tempranamente sus trayectorias escolares, presentarían problemas para dar continuidad a sus aprendizajes escolares (Julio, 2017). La medición de un autoinforme desde los/as propios/as estudiantes puede aportar a visualizar de manera temprana las voces y perspectivas críticas de los y las estudiantes hacia esfuerzos de mejora escolar (Fredricks \& McColskey, 2012).

En base a eso, se destaca la existencia de instrumentos de medición de Compromiso Escolar y de Factores Contextuales que influyen en el contexto chileno (Lara et al., 2018). Los resultados de los análisis psicométricos del instrumento de mediación del Compromiso escolar desarrollado por Lara et al. (2018), indican que el modelo de tres factores correlacionados da cuenta que el compromiso afectivo, cognitivo y conductual son distintos procesos, aunque fuertemente relacionados. Además, se afirma que el instrumento presentó una buena consistencia interna en cada una de las tres dimensiones, así como en la escala general (Lara et al., 2018). Complementariamente, el estudio realizado por Miranda-Zapata et al. (2018) aporta evidencia a la validez del modelo teórico que relaciona de forma directa los Factores Contextuales (familia, profesorado y pares) con el Compromiso Escolar (afectivo, cognitivo y conductual), y este último, a su vez, con el rendimiento escolar y la asistencia a clases; concluyendo que el Compromiso Escolar es una variable importante, que posee un efecto directo sobre las variables dependientes señaladas $\mathrm{y}$ de su rol mediador con los Factores Contextuales.

A partir de todo lo expuesto, el objetivo del presente estudio es adaptar y validar los instrumentos de medición del Compromiso Escolar y Factores Contextuales a niveles inferiores para los que fueron creados, concretamente, quinto y sexto de educación básica, posibilitando de este modo avanzar hacia una medición temprana del Compromiso Escolar y Factores Contextuales durante las trayectorias educativas de los y las estudiantes de Chile. Se espera que los instrumentos adaptados a esta nueva población de estudiantes presenten adecuados índices psicométricos.

La necesidad de ajustar los instrumentos de Compromiso Escolar y de Factores Contextuales, se fundamentan en tres ideas centrales: 1) Avanzar hacia un sistema de alerta temprana en 
Chile mediante la medición del Compromiso Escolar y Factores Contextuales (Lara et al., 2018), favoreciendo la generación de indicadores sociales y educativos capaces de ofrecer información válida para la toma de decisiones basado la percepción de los y las estudiantes. 2) Retroalimentar a la política pública educativa chilena, apoyando la labor preventiva de los gobiernos locales y comunidades educativas en su función del derecho a la educación y a las trayectorias educativas positivas de todos y todas las estudiantes. 3) La inexistencia hasta la fecha de instrumentos válidos y fiables, de medición de Compromiso Escolar y Factores Contextuales influyentes en el Compromiso Escolar, adaptado a los cursos de quinto y sexto de la educación básica en Chile.

\section{Método}

\section{Participantes}

En el estudio participaron 420 estudiantes que cursaban quinto $(n=204)$ y sexto $(n=216)$ de educación básica escolarizados en cinco escuelas municipales de tres regiones chilenas (Araucanía, Bernardo O' Higgins y Antofagasta). Las edades estuvieron comprendidas entre los 9 y 14 años $(M=10.91, D E=0.77)$, el $53.1 \%$ fueron niños $(n=223)$ y el $46.9 \%$ niñas $(n=197)$.

\section{Instrumentos}

Cuestionario Compromiso Escolar: Instrumento elaborado por Lara et al. (2018). Consistente en 29 ítems con una escala de respuesta tipo Likert de 5 puntos, orientado a evaluar los tres tipos de compromiso escolar: afectivo (10 ítems), cognitivo (12 ítems) y conductual (7 ítems). Cuenta con evidencia a favor de la validez estructural, con aceptables niveles de ajuste del modelo a los datos $\left(\mathrm{Chi}^{2}=548.52 ; g l=374 ; \mathrm{RMSEA}=.050 ; \mathrm{CFI}=.94\right.$; TLI=.93) y adecuados niveles de confiabilidad, con valores de alfa de Cronbach de .83 para la dimensión afectiva, .86 para la dimensión conductual y .87 para la dimensión cognitiva, mientras que la fiabilidad de la escala total es de .95 (Lara et al., 2018).

Cuestionario Factores Contextuales: Instrumento registrado por Universidad de la Frontera y Universidad Autónoma de Chile
(2016). Consistente en 18 ítems con una escala de respuesta tipo Likert de 5 puntos, los cuales evalúan los factores contextuales: familia (3 ítems), profesorado (8 ítems) y pares (7 ítems). Cuenta con evidencia a favor de la validez estructural, con aceptables niveles de ajuste del modelo a los datos $\left(\mathrm{Chi}^{2}=263.01 ; \quad g l=132\right.$; RMSEA=.070; CFI=.92; TLI=.91) y adecuados niveles de confiabilidad, con valores de alfa de Cronbach de .69 para familia, .83 para profesorado y .83 para pares, mientras que la fiabilidad de la escala total es de .88 (MirandaZapata et al., 2018).

\section{Procedimiento}

Previo a la recogida de datos en los centros, se obtuvieron los permisos de las escuelas, el consentimiento informado firmado por los/as representantes legales de los/as participantes, y el asentimiento firmado de los los/as estudiantes. Los instrumentos fueron complementados de forma colectiva en los centros educativos en horario lectivo, con la presencia de un miembro del estudio debidamente capacitado para supervisar la aplicación. La aplicación se realizó de forma online en las salas de computación de los centros educativos, a través de una plataforma informatizada desarrollada para tal efecto (Saracostti, Lara, \& Miranda-Zapata, 2019), con una duración aproximada de entre 30 y 40 minutos. Complementariamente, se solicitó a las comunidades educativas la edad, el promedio de notas y el porcentaje de asistencia del semestre de cada estudiante. El estudio recibió la aprobación del Comité de Ética de la Universidad de la Frontera.

\section{Plan de análisis}

Dado que se conoce la estructura factorial de ambos cuestionarios, la estrategia de validación de la estructura de los cuestionarios se ha centrado en la realización de Análisis Factoriales Confirmatorios (AFC), con el objetivo de ratificar si la estructura de los cuestionarios desarrollados para cursos superiores se adecua a cursos inferiores (quinto y sexto básico).

Los AFC han sido realizados mediante el programa Mplus 7.3, sobre la matriz de correlaciones policóricas, con el método de estimación de Cuadrados Mínimos No Ponderados 
Robusto (ULSMV). Para los índices de adecuación AFC se consideraron los puntos de cortes establecidos por Arbuckle (2011), incluyendo el Error Cuadrático Medio de Aproximación (Root Mean Square Error of Approximation, RMSEA <.08), el Índice de Ajuste Comparativo (Complex Fix Index, CFI>.90), y el Índice de Tucker-Lewis (Tucker-Lewis Index, TLI>.90).

Dada la naturaleza ordinal de los datos, la estimación de la consistencia interna de los instrumentos se ha realizado mediante el índice Omega de McDonald, utilizando el programa FACTOR que se basa en la matriz de correlaciones policóricas, tanto para el total de las escalas como para cada una de sus dimensiones. Complementariamente, se presenta el alfa de Cronbach, a pesar de estar basado en la matriz de correlaciones de Pearson, por ser el índice más utilizado en las ciencias sociales.

Para la validez externa (predictiva), se realizaron análisis de correlaciones bivariadas entre las puntuaciones de los cuestionarios y los criterios asistencia y promedio de notas, recogidos al finalizar el semestre en el que se realizó la recogida de datos.

\section{Resultados}

\section{Adaptación de los cuestionarios}

En primer lugar, se realizó la adaptación lingüística de los ítems que componen los cuestionarios de Compromiso escolar y Factores contextuales. Para ello, se realizaron cinco entrevistas a expertos/as en el área de educación, quienes revisaron los ítems y elaboraron sugerencias de adaptación lingüística. En segundo lugar, una vez unificados los cambios propuestos por los expertos y las expertas, estos fueron revisados mediante grupos de discusión con profesionales de la educación (5 grupos compuestos por 5 profesionales) y por seis grupos de discusión con estudiantes (uno por nivel compuesto por 6 estudiantes en cada una de las regiones participantes). Todos los cambios fueron revisados por el equipo de investigación, el $61 \%$ de los ítems del cuestionario de Compromiso Escolar y el 50\% de los ítems del cuestionario de Factores Contextuales fueron modificados en su redacción para ser más entendibles por los y las estudiantes, con ajustes lingüísticos para favorecer su pertinencia y compresión. En el anexo se presentan las versiones finales de los cuestionarios. A continuación, se presentan de forma separada los resultados del proceso de validación para cada cuestionario.

\section{Validación Cuestionario de Compromiso Escolar}

\section{Estructura factorial}

Se llevó a cabo el AFC para comprobar si los datos se ajustaban a la estructura de un modelo de tres factores correlaciones, siendo así que el Compromiso Escolar se compone de los tres factores esperados (Conductual, Afectivo y Cognitivo) de forma correlacionada (Modelo de tres factores correlacionados). Los resultados del AFC muestran un aceptable ajuste de los datos al modelo $\left(\mathrm{Chi}^{2}=733.122 ; g l=374 ; \mathrm{RMSEA}=.048\right.$; $\mathrm{CFI}=.930$; $\mathrm{TLI}=.924)$. Las saturaciones factoriales estandarizadas oscilaron entre .421 y .850 . En cuanto a las correlaciones entre los factores, la correlación entre el factor afectivo y Cognitivo fue de .678, entre el afectivo y el Conductual de .421 y entre el Cognitivo y el Conductual de .514 .

\section{Fiabilidad}

Los análisis de fiabilidad mostraron que la tanto la escala completa, como las dimensiones que la componen, muestran una adecuada consistencia interna (Tabla 1).

Tabla 1. Consistencia interna del cuestionario

\begin{tabular}{lcc}
\multicolumn{3}{c}{ Compromiso Escolar } \\
\hline Dimensión & $\alpha$ Cronbach & Omega \\
\hline Afectiva & .844 & .880 \\
Conductual & .680 & .846 \\
Cognitiva & .880 & .905 \\
Total & .902 & .925 \\
\hline Nota. ${ }^{* *} p<.01$ & &
\end{tabular}

\section{Validez predictiva}

Finalmente, se analizaron las correlaciones bivariadas entre las puntuaciones de los tres componentes del Compromiso Escolar con los promedios de notas y los porcentajes de asistencia. Como se observa en la Tabla 2, las correlaciones fueron significativas en todos los casos, excepto entre el componente afectivo y los porcentajes de asistencia de los/as estudiantes, indicando, por tanto, que un mayor compromiso en todos los factores se asocia con un promedio de 
notas superior, así como una mayor asistencia a clase en el caso de los componentes Cognitivo y Conductual, no así del Afectivo.

Tabla 2. Correlaciones entre los componentes del Compromiso Escolar y el promedio de notas y la asistencia

\begin{tabular}{llc}
\hline & Notas & Asistencia \\
\hline Compromiso Afectivo & $.223 * *$ & .078 \\
Compromiso Conductual & $.439 * *$ & $.171 * *$ \\
Compromiso Cognitivo & $.346 * *$ & $.127 * *$ \\
\hline Nota. ${ }^{*} p<.01$ & &
\end{tabular}

\section{Validación Cuestionario de Factores Contextuales}

\section{Análisis de la estructura factorial}

Se llevó a cabo el AFC para comprobar si los datos se ajustaban a la estructura de un modelo de tres factores correlaciones: los Factores Contextuales se componen de tres factores que representan el apoyo de familia, profesorado y pares, que se encuentran correlacionados (Modelo de tres factores correlacionados). Los resultados del AFC muestran un buen ajuste de los datos al modelo $\left(\mathrm{Chi}^{2}=296.969 ; g l=132 ; \mathrm{RMSEA}=.055\right.$; $\mathrm{CFI}=.957$; $\mathrm{TLI}=.951)$. Las saturaciones factoriales estandarizadas oscilaron entre .633 y .816. En relación a las correlaciones entre factores, la correlación entre el factor profesorado y familia fue de .674 , entre pares y familia de .413 y entre profesorado y pares de .584 .

\section{Análisis de fiabilidad}

Los análisis de fiabilidad mostraron que la tanto la escala completa, como las dimensiones que la componen, muestran una adecuada consistencia interna (Tabla 3).

Tabla 3. Consistencia interna del cuestionario Factores Contextuales

\begin{tabular}{lcc}
\hline Dimensión & $\alpha$ Cronbach & Omega \\
\hline Familia & .654 & .762 \\
Profesores & .844 & .883 \\
Pares & .861 & .886 \\
Total & .884 & .911 \\
\hline Nota. ${ }^{* *} p<.01$ & &
\end{tabular}

\section{Validez predictiva}

Finalmente, se analizaron las correlaciones bivariadas entre las puntuaciones de los tres Factores Contextuales y los promedios de notas y el porcentaje de asistencia. Como se observa en la
Tabla 4, las correlaciones fueron significativas en el caso de los promedios de notas, indicando por lo tanto que las mayores puntuaciones en los tres contextos (familia, pares y profesorado) se asocian con un mejor promedio de notas, pero no a una mayor asistencia a clase.

Tabla 4. Correlaciones entre los Factores

Contextuales y el promedio de notas y la asistencia

\begin{tabular}{llc}
\hline & Notas & Asistencia \\
\hline Familia & $.251^{* *}$ & .090 \\
Profesores & $.293^{* *}$ & .066 \\
Pares & $.127^{* *}$ & .072 \\
\hline \multicolumn{1}{c}{ Nota. ${ }^{* *} p<.01$} & &
\end{tabular}

\section{Discusión}

La literatura sugiere que el Compromiso Escolar sería un constructo multidimensional (Fredricks et al., 2004), una metaconstrucción (Fredricks et al., 2004; Kahu, 2013), maleable y abierto a condiciones contextuales (Furlong et al., 2008); además de ser un predictor significativo del rendimiento académico (Appleton et al., 2008; Hazel et al., 2013) y de la deserción escolar (Wang \& Eccles, 2012). La medición de Compromiso Escolar se correlaciona positivamente con el rendimiento y negativamente con la probabilidad de abandonar la escuela (Fredricks et al., 2012).

A partir de los resultados obtenidos en este estudio, se puede concluir que se cuenta con un instrumento que mide de forma adecuada el Compromiso Escolar en estudiantes de quinto y sexto de enseñanza básica. Más en detalle, el resultado del análisis de la estructura factorial demostró un aceptable ajuste de los datos a la estructura de un modelo de los tres factores (afectivo, conductual y cognitivo) correlacionados, coincidente con la configuración teórica del concepto (Fredricks et al., 2004; Lara et al., 2018).

Con respecto a los análisis de fiabilidad del cuestionario mostraron una adecuada consistencia interna de la escala completa y de las dimensiones que la componen. Al mismo tiempo, los análisis de validez predictiva demostraron que las correlaciones entre las puntuaciones de los tres componentes del Compromiso Escolar, con los promedios de notas y los porcentajes de asistencia 
fueron significativas en todos los casos, excepto entre el Componente Afectivo y los porcentajes de asistencia de los/as estudiantes.

Además, se puede concluir que se cuenta con un instrumento que mide de forma adecuada los Factores Contextuales en estudiantes de quinto y sexto de enseñanza básica. En cuanto a los AFC, se demostró un buen ajuste de los datos al modelo de tres factores correlaciones, específicamente entre los factores de relación con familia, profesorado y pares, que teóricamente configuran este constructo (Ansong et al., 2017; Gutiérrez et al., 2017; Miranda-Zapata et al., 2018; RodríguezFernández et al., 2016). Además, los análisis de fiabilidad mostraron que la tanto la escala completa, como las dimensiones que la componen, muestran una adecuada consistencia interna.

En cuanto a la validez predictiva del instrumento de medición de Factores Contextuales, se realizaron correlaciones bivariadas entre los tres componentes (familia, profesorado y pares), notas y asistencia. Estas fueron significativas en el caso de los promedios de notas, debido a que las mayores puntuaciones en los tres contextos se asocian con un mejor promedio de notas, pero no con una mayor asistencia a clase. Por lo cual, la asistencia no tendría una correlación significativa con los Factores Contextuales. Cabe decir que durante la infancia es donde surge la autoevaluación mediante comparaciones sociales, volviéndose especialmente importante la internalización de las opiniones de los otros significativos como familia, profesorado y pares (Milicic \& Marchant, 2020).

Los instrumentos de medición del Compromiso Escolar y de Factores Contextuales adaptados y validados para los cursos de quinto y sexto básico en Chile posibilita a las comunidades escolares tomar decisiones informadas. De este modo, permite la identificación de aquellos y aquellas estudiantes en riesgo mediante reportes de valoraciones bajas en los ítems que componen los instrumentos, $\mathrm{y}$, específicamente, a que factores se debe. Cabe mencionar que la identificación temprana de indicadores de riesgo podría fortalecer a la promoción de Compromiso Escolar y trayectorias educativas exitosas en edad temprana (Ladd et al., 2009). Por el contrario, también posibilita conocer las fortalezas con la que los y las estudiantes cuentas en su proceso educativo y su contexto escolar. Los/as estudiantes que auto reporten un alto Compromiso Escolar podría indicar que poseen mayores probabilidades de tener una trayectoria educativa exitosa (Furlong et al., 2008) y menor propensión al abandono escolar (Appleton et al., 2008; Fredricks et al., 2004; Li, Gao, \& Sha, 2020; Rumberger et al., 2012; Van Dijk, 2012).

Cómo limitación se puede mencionar que el estudio contempló la participación de 420 estudiantes de quinto y sexto básico de la educación chilena, abarcando una muestra representativa acotada de la población en estudio y podría dejar fuera del alcance de los/as investigadores/as algunas temáticas emergentes relacionados al tema de estudio.

En cuanto a las fortalezas, es importante mencionar que el presente artículo permite profundizar en la discusión acerca de la medición del Compromiso Escolar y Factores Contextuales a través de la autopercepción de niños y niñas en Chile. Se valora como positivo el ajuste del instrumento de medición de Compromiso Escolar y Factores Contextuales para niños y niñas de cursos menores (quinto y sexto básico) de la educación chilena, debido a que representan un segmento de la población que requiere de una temprana protección, acompañamiento y apoyo en su proceso educativo.

Se considera que un sistema de alerta temprana de los/as estudiantes con riesgo de desertar, formarían parte de un proceso integral, que se basa en investigación sobre los predictores académicos y de comportamiento del abandono escolar (Bailey, Dailey, Schanfield, \& Zuber, 2017). En base a eso, el Compromiso Escolar y Factores Contextuales asociados, han demostrado ser indicadores tempranos y válidos para la prevención del abandono escolar (Miranda-Zapata et al., 2018). Siendo la infancia el período más relevante para la toma de decisiones personales respecto a los proyectos de vida en formación (Milicic et al., 2020), se hace necesario comenzar a situar el foco en la educación inicial en Chile, de manera predecir resultados educativos a largo plazo (Ladd et al., 2009), aunque no todo sería controlable, ya que en el período temprano de la adolescencia se presentan cambios personales que impactan en el entorno social más importante, 
especialmente durante la transición a la escuela secundaria (Białecka-Pikul, Stępień-Nycz, Sikorska, Topolewska-Siedzik, \& Cieciuch, 2019).

Además, la profundización en el concepto de Compromiso Escolar podría ayudar al Estado y al tercer sector, a tomar mejores decisiones frente al diseño y orientación de las políticas públicas en materias educativas (Fredricks et al., 2012). A raíz del presente estudio, se visualiza como necesario avanzar hacia la investigación que pueda identificar, probar y validar estrategias biopsicosociales de promoción del Compromiso Escolar y Factores Contextuales, que faciliten el transito educativo desde la educación primaria a la secundaria en Chile. Un posible foco de la identificación de las estrategias podría ser el desarrollo de habilidades sociales en estudiantes, teniendo en cuenta que estas habilidades se relacionarían, al igual como el Compromiso escolar, con los logros escolares (Salas, Asún, \& Zúñiga, 2020).

\section{Financiamiento}

Este artículo ha recibido el apoyo del proyecto CORFO 18ISV-93400 financiado por el Ministerio de Economía, Fomento y Turismo, Chile y por el proyecto FONDEF IT $19 \mathrm{I} 0012$ financiado por la Comisión Nacional de Investigación Científica y Tecnológica CONICYT, Chile.

\section{Referencias}

Abraham, M., \& Lavín, S. (2017). La deserción escolar: un desafío pedagógico y social. Paulo Freire. Revista de Pedagogía Crítica, (6), 147-168. doi:10.25074/07195532.6.485

Alrashidi, O., Phan, H., \& Ngu, B. (2016). Academic engagement: An overview of Its definitions, dimensions, and major conceptualizations. International Education Studies, 9(12), 41-52. doi:10.5539/ies.v9n12p41

Ansong, D., Okumu, M., Bowen, G., Walker, A., \& Eisensmith, S. (2017). The role of parent, classmate, and teacher support in student engagement: Evidence from Ghana. International Journal of Educational Development, 54, 51-58. doi:10.1016/j.ijedudev.2017.03.010
Appleton, J. J., Christenson, S. L., \& Furlong, M. J. (2008). Student engagement with school: Critical, conceptual, and methodological issues of the construct. Psychology in the Schools, 45, 369-386. doi:10.1002/pits.20303

Arbuckle, J. L. (2011). IBM SPSS Amos 20 user's guide. Armonk, NY, EE.UU.: Amos Development Corporation.

Archambault, I., \& Vandenbossche-Makombo, J. (2014). Validation de l'échelle des dimensions de l'engagement scolaire (ÉDES) chez les élèves du primaire. Revue canadienne des sciences $d u$ comportement, 46(2), 275288. doi:10.1037/a0031951

Bailey, B., Dailey, D., Schanfield, M., \& Zuber, T. (2017). Early warning intervention and monitoring system implementation guide: For use with the Michigan data hubs early warning system tool. American Institutes for Research. Michigan Department of Education.

Białecka-Pikul, M., Stępień-Nycz, M., Sikorska, I., Topolewska-Siedzik, E., \& Cieciuch, J. (2019). Change and consistency of selfesteem in early and middle adolescence in the context of school transition. Journal of Youth and Adolescence, 48, 1605-1618.

doi:10.1007/s10964-019-01041-y

Bond, M., Buntins, K., Bedenlier, S., ZawackiRichter, O., \& Kerres, M. (Eds.). (2020). Systematic Reviews in Educational Research: Methodology, Perspectives and Application. doi:10.1007/978-3-658-27602-7

Centro de Estudios del Ministerio de Educación (2020). Medición de la exclusión escolar en Chile. (Documento de trabajo $\mathrm{N}^{\circ}$ 20). Santiago, Chile.

Centro de Investigación Avanzada en Educación, CIAE. (2019). Caracterización cuantitativa de trayectorias escolares en jóvenes con algún grado de exclusión educacional: Informe Técnico. Santiago, Chile. Recuperado de http://ciae.uchile.cl/download. php?file=noticias/00_1552671019.pdf

Forero, I., \& Lugo, G. (2017). Diseño de un plan de gestión temprana a través del desarrollo de actividades motivadoras para disminuir la deserción escolar en la institución educativa la Magdalena. (Tesis de Magíster inédita). Universidad Libre, Colombia, Bogotá D.C. 
Fredricks, J. A., \& McColskey, W. (2012). The measurement of student engagement: A comparative analysis of various methods and student self-report Instruments. En Christenson S., Reschly A., y Wylie C. (eds) Handbook of Research on Student Engagement (pp. 763-782). doi:10.1007/978-1-4614-2018-7_37

Fredricks, J. A., Blumenfeld, P. C., \& Paris, A. H. (2004). School engagement: Potential of the concept, state of the evidence. Review of Educational Research, 74(1), 59-109. doi:10.3102/00346543074001059

Furlong, M. J., \& Christenson, S. L. (2008). Engaging students at school and with learning: A relevant construct for all students. Psychology in the Schools, 45(5), 365-368. doi:10.1002/pits.20302

García, B. (2016). Indicadores de abandono escolar temprano: Un marco para la reflexión sobre estrategias de mejora. Perfiles Educativos, 38(154), 191-213. Recuperado de:

http://www.scielo.org.mx/scielo.php?script=sc i_arttext\&pid=S0185-

26982016000400011\&lng=es\&tlng=es

Gutiérrez, M., Tomás, J., Romero I., \& Barrica, J. (2017). Apoyo social percibido, implicación escolar y satisfacción con la escuela. Revista de Psicodidáctica, 22(2), 111-117. doi:10.1016/j.psicod.2017.01.001

Hazel, C. E., Vazirabadi, G. E., \& Gallagher, J. (2013). Measuring aspirations, belonging, and productivity in secondary students: Validation of the Student School Engagement Measure. Psychology in the Schools, 50(7), 689-704. doi:10.1002/pits.21703

Hazel, C. E., Wonner, R., \& Jack, C. (2008). School engagement: What is it and how to get more of it. Paper presented at the 33th annual convention of the Colorado Society of School Psychologists, Avon, CO.

Julio, C. (2017). Educandos fuera del sistema escolar: Derecho conculcado y un compromiso pendiente en la Educación Especial Chilena. Revista Latinoamericana de Educación Inclusiva, 11(1), 109-129. Recuperado de http://repositoriocdpd.net:8080/handle/123456 $789 / 1845$
Kahu, E. R. (2013). Framing Student Engagement in Higher Education. Journal Studies in Higher Education, 38(5), 758-773. doi:10.1080/03075079.2011.598505

Ladd, G. W., \& Dinella, L. M. (2009). Continuity and change in early school engagement: Predictive of children's achievement trajectories from first to eighth grade? Journal of Educational Psychology, 101(1), 190-206. doi:10.1037/a0013153

Lam, S.-F., Jimerson, S., Wong, B., Kikas, E., Shin, H., Veiga, F. H., ... Zollneritsch, J. (2014). Understanding and measuring student engagement in school: The results of an international study from 12 countries. School Psychology Quarterly, 29(2), 213-232. doi:10.1037/spq0000057

Lara, L., Saracostti, M., Navarro, J.-J., de-Toro, X., Miranda-Zapata, E., Trigger, J. M., \& Fuster, J. (2018). Compromiso escolar: Desarrollo y validación de un instrumento. Revista Mexicana de Psicología, 35(1), 52-62.

Li, W., Gao, W., \& Sha, J. (2020). Perceived teacher autonomy support and school engagement of Tibetan students in elementary and middle dchools: Mediating effect of selfefficacy and academic emotions. Frontiers in Psychology, 11(50). doi:10.3389/fpsyg. 2020.00050

Milicic, N., \& Marchant, T. (2020). Educación emocional en el sistema escolar chileno: Un desafío pendiente. En Corvera M.T. y Gonzalo Muñoz, G. (Eds.), Horizontes y propuestas para transformar el sistema educativo chileno (52-77). Santiago, Chile: Ediciones Biblioteca del Congreso Nacional de Chile.

Miranda-Zapata, E., Lara, L., Navarro, J. J., Saracostti, M., \& De Toro, X. (2018). Modelización del efecto del Compromiso Escolar sobre la asistencia a clases y el rendimiento escolar. Revista de Psicodidáctica, 23(2), 102-109. doi:10.1016/j.psicod.2018.02.003

Molinari, A., \& Sánchez-Rosas, J. (2018). Compromiso comportamental: Propuestas para la evaluación de participación social académica. Revista Contextos de Educación, 20(25), 99-109. 
Rodríguez-Fernández, A., Ramos-Díaz, E., Fernández-Zabala, A., Goñi, E., Esnaola, I., \& Goñi, A. (2016). Contextual and psychological variables in a descriptive model of subjective well-being and school engagement. International Journal of Clinical and Health Psychology, 16(2), 166-174. doi:10.1016/j.ijchp.2016.01.003

Rumberger, R. W., \& Lim, A. S. (2008). Why students drop out of school: A review of 25 years of research. California Dropout Research Project Report $\mathrm{N}^{\circ}$ 15. Santa Bárbara: University of California.

Rumberger, R. W., \& Rotermund, S. (2012). The relationship between engagement and high school dropout. En Christenson S., Reschly A., y Wylie C. (eds) Handbook of Research on Student Engagement (pp. 491-513). doi:10.1007/978-1-4614-2018-7_24

Rumberger, R. W., Addis, H., Allensworth, E., Balfanz, R., Duardo, D., \& Dynarski, M. (2017). Preventing dropout in secondary schools. Washington, DC: National Center for Education Evaluation and Regional Assistance (NCEE), Institute of Education Sciences, U.S. Department of Education. Recuperado de https://whatworks.ed.gov

Sagar, R., \& Mehta, M. (Eds.). (2018). A practical approach to cognitive behaviour therapy for adolescents. Springer India. doi:10.1007/978-81-322-2241-5

Salas, P., Asún, R., \& Zúñiga, C. (2020). Construcción de un Cuestionario de Habilidades Sociales para el Contexto Académico (CHS-A). Revista Iberoamericana de Diagnóstico y Evaluación - e Avaliação Psicológica, 55(2), 89-105. doi:10.21865/RIDEP55.2.07

Sandoval-Muñoz, M. J., Mayorga-Muñoz, C., Elgueta-Sepúlveda, H., Soto-Higuera, A., Viveros-Lopomo, J., \& Riquelme-Sandoval, S. (2018). Compromiso y motivación escolar: Una discusión conceptual. Revista Educación, 42(2). doi:10.15517/REVEDU.V42I2.23471

Saracostti, M., Lara, L., \& Miranza-Zapata, E. (2019). Plataforma tecnológica de evaluación del compromiso escolar, factores de contexto y reporte de resultados online: Breve reporte técnico. Electronic Journal of Research in Educational Psychology, 17(1), 193-212. doi:10.25115/ejrep.v17i47.2049

Shernoff, D. J., Ruzek, E. A., \& Sinha, S. (2017). The influence of the high school classroom environment on learning as mediated by student engagement. School Psychology International, 38(2), 201-218. doi:10.1177/0143034316666413

Shernoff, D. J. (2013). Optimal learning environments to promote student engagement. Springer Science + Business Media. doi:10.1007/978-1-4614-7089-2

Shernoff, D. J. (2012). Engagement and positive youth development: Creating optimal learning environments. En K. R. Harris, S. Graham, T. Urdan, S. Graham, J. M. Royer, y M. Zeidner (Eds.), APA handbooks in psychology®. APA educational psychology handbook, Vol. 2. Individual differences and cultural and contextual factors (p. 195-220). American Psychological Association. doi:10.1037/13274-008

Pérez-Salas, C., Sirlopú, D., Cobo-Rendón, R., \& Awad, A. (2018). Análisis bifactorial de la Escala de Participación Escolar en una muestra de estudiantes Chilenos. Revista Iberoamericana de Diagnóstico y Evaluación - e Avaliação Psicológica, 52(3), 27-39. doi:10.21865/RIDEP52.3.03

Van Dijk, S. (2012). La política pública para abatir el abandono escolar y las voces de los niños, sus tutores y sus maestros. Revista mexicana de investigación educativa, 17(52), 115-139. Recuperado de: https://www.redalyc.org/articulo.oa?id=140/1 4023076006

Veiga, F. H. (2013). Envolvimento dos alunos na escola: Elaboração de uma nova escala de avaliação. International Journal of Developmental and Educational Psychology, 1(1), 441-450. Recuperado de: https://www.redalyc.org/articulo.oa?id=3498/ 349852058036

Wang, M.-T., \& Eccles, J. S. (2012). Adolescent behavioral, emotional, and cognitive engagement trajectories in school and their differential relations to educational success. Journal of Research on Adolescence, 22(1), 3139. doi:10.1111/j.1532-7795.2011.00753.x

Zaff, J., Donlan, A., Gunning, A., Anderson, S., McDermott, E., \& Sedaca, M. (2017). Factors 
that promote high school graduation: A review of the literature. Educational Psychology Review, 29(3), 447-476.

doi:10.1007/s10648-016-9363-5

Zurita, Ú. (2011). Los desafíos del derecho a la educación en México a propósito de la participación social y la violencia escolar. Revista Mexicana de Investigación Educativa, 16(48), 131-158. Recuperado de: https://www.redalyc.org/articulo.oa?id=140/1 4015561007 
Anexo 1.

\section{Cuestionario Compromiso Escolar}

\begin{tabular}{|c|c|c|}
\hline Dimensiones & $\mathbf{N}^{\mathbf{0}}$ & Ítems \\
\hline Afectivo & 1 & Siento que soy parte de esta escuela \\
\hline Cognitivo & 2 & Antes de una prueba, reviso cómo estudiar la materia \\
\hline Conductual & 3 & Me escapo de clases o me corro de clases (r) \\
\hline Conductual & 4 & Salgo sin pedir permiso de la sala (r) \\
\hline Afectivo & 5 & Puedo ser yo mismo(a) en esta escuela \\
\hline Cognitivo & 6 & $\begin{array}{l}\text { Uso distintos recursos (como internet o libros) para entender mejor la información } \\
\text { entregada por el profesor (a) }\end{array}$ \\
\hline Afectivo & 7 & La mayoría de las cosas que aprendo en la escuela sirven para mi vida \\
\hline Afectivo & 8 & $\begin{array}{l}\text { La mayoría de los/as profesores(as) se preocupan de que la materia que aprendamos } \\
\text { sirva para mi vida }\end{array}$ \\
\hline Conductual & 9 & Llego atrasado(a) a clases (r) \\
\hline Cognitivo & 10 & Cuando estoy haciendo alguna actividad, me preocupo de entender lo más posible \\
\hline Conductual & 11 & Mis apoderados han sido citados por mi mala conducta $(r)$ \\
\hline Afectivo & 12 & Siento orgullo de estar en esta escuela \\
\hline Cognitivo & 13 & Después de una prueba, reviso si las respuestas fueron las correctas \\
\hline Cognitivo & 14 & Sé cómo cambiar mi manera de estudiar para mejorar y obtener mejores notas \\
\hline Afectivo & 15 & Para mí es muy importante lo que hacemos en la escuela \\
\hline Conductual & 16 & Me porto bien en clases \\
\hline Cognitivo & 17 & $\begin{array}{l}\text { Cuando comienzo una tarea, recuerdo lo que he aprendido de la materia porque eso me } \\
\text { ayuda a comprender mejor }\end{array}$ \\
\hline Cognitivo & 18 & Cuando estudio, anoto palabras nuevas, dudas o ideas importantes \\
\hline Afectivo & 19 & Me tratan con respeto en esta escuela \\
\hline Cognitivo & 20 & Para mí es importante lograr entender bien las tareas y la materia \\
\hline Cognitivo & 21 & $\begin{array}{l}\text { Sé cómo utilizar diferentes formas de estudio para realizar bien mis tareas (como, por } \\
\text { ejemplo, planificar el trabajo, repasar la materia, estudiar en grupos, etc.) }\end{array}$ \\
\hline Afectivo & 22 & Lo que aprendo en clases es importante para lograr lo que quiero en el futuro \\
\hline Conductual & 23 & Peleo con mis compañeros en la sala $(r)$ \\
\hline Cognitivo & 24 & Después de terminar mis tareas reviso si están bien \\
\hline Cognitivo & 25 & Cuando termino una tarea, pienso si lo hice bien \\
\hline Cognitivo & 26 & Pongo atención a los comentarios que los/as profesores hacen sobre mis trabajos \\
\hline Afectivo & 27 & Siento que soy importante para la escuela \\
\hline Conductual & 28 & Me mandan a la oficina del director o del inspector general por mi mala conducta $(r)$ \\
\hline Afectivo & 29 & Me siento bien en esta escuela \\
\hline
\end{tabular}

Nota. $\mathrm{r}=1$ tem reverso. 
Anexo 2.

\section{Cuestionario Factores Contextuales}

\begin{tabular}{cll}
\hline Dimensiones & $\mathrm{N}^{\mathbf{o}}$ & Ítem \\
\hline Familia & 1 & Hablo con mi familia sobre lo que hago en la escuela \\
& 2 & Mis padres o apoderados(as) me animan a trabajar bien en la escuela \\
Profesores & 3 & Cuando tengo un problema, recibo ayuda de mi familia \\
& 5 & Mis profesores(as) quieren que aprenda mucho. \\
& 6 & Cuando tengo un problema, recibo ayuda de algún(a) profesor(a). \\
7 & Los/as profesores(as) se interesan por mí y me ayudan si tengo problemas para \\
& hacer las tareas. \\
& 9 & Me llevo bien con mis profesores(as). \\
& 10 & Los/as profesores(as) se preocupan de mí no sólo como estudiante sino también \\
& En mi escuela, los/as profesores(as) y otros(as) adultos(as) tratan a todos los/as \\
& 11 & estudiantes con respeto. \\
12 & En esta escuela, se valora la participación y la opinión de todos(as) \\
13 & Puedo confiar en mis compañeros(as). \\
14 & Mis compañeros(as) de la escuela son importantes para mí. \\
15 & Me llevo bien con mis compañeros(as) de curso \\
16 & Siento que soy importante para mis compañeros(as) de la escuela \\
17 & En mi escuela, al menos un(a) compañeros(a) me apoya con las tareas difíciles \\
18 & Cuando no entiendo algo, mis compañeros(as) me explican
\end{tabular}

